\section{Prevalência e fatores associados a indicadores negativos de saúde mental em adolescentes estudantes do ensino médio em Pernambuco, Brasil}

\section{The prevalence of and factors associated with negative mental health indicators in adolescents attending high school in the Brazilian State of Pernambuco}

Priscila Diniz de Carvalho 1

Mauro Virgilio Gomes de Barros 2

Carla Meneses Santos 3

Edilânea Nunes Melo 4

Nilma Kelly Ribeiro de Oliveira 5

Rodrigo Antunes Lima 6

1-2 Universidade de Pernambuco. Faculdade de Odontologia. Programa de Pós-graduação em Hebiatria. Grupo de Pesquisa em Hebiatria. Recife - PE, Brasil. CEP: 54753-901.

E-mail: prifermeira@hotmail.com

3-6 Universidade de Pernambuco. Escola Superior de Educação Física. Programa Associado de Pós-graduação em Educação Física UPE/UFPB. Grupo de Pesquisa em Estilos de Vida e Saúde. Recife - PE, Brasil.

\section{Resumo}

Objetivos: identificar a prevalência e fatores associados a indicadores negativos de saúde mental em adolescentes.

Métodos: utilizou-se um questionário previamente validado para coletar dados de 4.207 adolescentes (14-19 anos) estudantes do ensino médio, selecionados através de amostragem por conglomerados em dois estágios.

Resultados: as prevalências de tristeza, sentimento de solidão, pensamento de suicídio, dificuldade para dormir devido à preocupação e planos de suicidio foram, respectivamente, 29,7\%, 15,8\%, $11,2 \%, 10,0 \%$ e 7,8\%. A prevalência de todos os indicadores foi superior entre as moças, sendo a tristeza o indicador mais prevalente. Identificou-se que a cor da pele não foi um fator associado à ocorrência dos indicadores negativos de saúde mental, enquanto não morar com os pais apresentou associação significativa com sentimento de solidão e pensamento em suicídio.

Conclusões: a prevalência de sentimento de solidão e de tristeza é alta e as moças representam um subgrupo de maior risco em relação a todos os indicadores negativos de saúde mental.

Palavras-chave Adolescente, Saúde mental, Suicídio, Solidão, Conduta de saúde 


\section{Introdução}

Estima-se que, em todo o mundo, aproximadamente $20 \%$ das crianças e adolescentes sofram de algum tipo de doença mental incapacitante, estando a ansiedade, depressão e outros distúrbios de humor entre os problemas mais comuns. ${ }^{1}$ Há evidências também que metade dos casos de desordens mentais, que se manifestam durante a vida, tem inicio na adolescência. $^{2}$

Crianças e adolescentes que sofrem com problemas de saúde mental são incapazes de utilizar apropriadamente os recursos para identificação e tratamento e, por conseguinte, terminam ainda mais expostos ao risco de abuso de álcool e outras drogas, baixo desempenho escolar, atividades violentas e criminosas, idealização e pensamento suicida. A cada ano, cerca de quatro milhões de adolescentes em todo o mundo tentam suicídio, sendo este a terceira causa de morte neste subgrupo populacional. ${ }^{3}$

Neste contexto, uma abordagem de investigação que vem sendo adotada é a análise de fatores pessoais e comportamentais associados aos indicadores de saúde mental, como os estudos conduzidos por Zhang et al. 4 e Panter-Brick et al. 5 que examinaram a associação entre estresse e saúde mental. Outros estudos focalizaram na inter-relação entre indicadores de saúde mental e fatores psicossociais, $5-7$ violência8-10 e condutas de risco à saúde. $6,11,12$

Em estudos epidemiológicos com adolescentes, a investigação de indicadores de saúde mental vem sendo realizada mediante monitoramento de variáveis como o sentimento de tristeza, a dificuldade de dormir devido à preocupação, o sentimento de solidão, a ideação suicida, e a relação destas com outros fatores relacionados à saúde. No estudo de Cheng et al.,13 conduzido com adolescentes chineses, os resultados evidenciaram que há associação significativa entre a exposição a situações de bullying e alguns indicadores de saúde mental. Xiong et al. ${ }^{14}$ realizaram um estudo sobre tendências suicidas entre estudantes do nível médio de cidades chinesas, observando-se que meninas apresentavam maior prevalência de pensamentos e planos de suicídio do que rapazes.

Page ${ }^{11}$ conduziu uma investigação com adolescentes africanos para descrever indicadores de saúde mental, uso de álcool e comportamento sexual na amostra, verificando que a exposição a comportamentos sexuais de risco tem associação com a ocorrência de indicadores negativos de saúde mental. Outro estudo realizado por Mahfoud 15 apresentou dados de tendência suicida em adolescentes libaneses, os resultados demonstraram que os adolescentes com pensamentos e planos de suicídios apresentaram maior prevalência de uso de substâncias psicotrópicas, de relato de maus tratos e de falta de apoio familiar.

No Brasil, estudo realizado com crianças e adolescentes de comunidade rural do município de Salvador, Estado da Bahia, evidenciou uma taxa de prevalência para um ou mais transtornos psiquiátricos que variou de $7 \%$ a $12,7 \% .^{3}$ Mas, investigações focalizando a análise da prevalência de exposição a indicadores de saúde mental em adolescentes brasileiros são escassos e até onde se tem conhecimento nenhum levantamento de abrangência estadual foi realizado. Assim, neste estudo, pretende-se identificar a prevalência e fatores associados a indicadores negativos de saúde mental em adolescentes estudantes do ensino médio no Estado de Pernambuco.

\section{Métodos}

Este estudo epidemiológico transversal foi desenvolvido como parte do projeto de pesquisa denominado "Estilos de vida e comportamentos de risco à saúde em estudantes do ensino médio no Estado de Pernambuco". O protocolo do estudo foi submetido ao Comitê de Ética em Pesquisa com seres humanos do Hospital Agamenon Magalhães, na cidade do Recife, em atendimento à indicação do Sistema Nacional de Ética na Pesquisa com seres humanos, tendo sido aprovado em julho de 2005.

A população alvo foi limitada aos adolescentes (14 a 19 anos), estudantes da rede pública estadual e do ensino médio residentes no Estado de Pernambuco. Considerando-se todas as dependências administrativas (federal, estadual, municipal e privada), os dados fornecidos pela Secretaria de Educação do Estado indicavam que, em 2006, os sujeitos matriculados na rede pública estadual representavam cerca de $80 \%$ do total de estudantes do ensino médio em Pernambuco. O dimensionamento da amostra foi efetuado de forma a atender os diversos objetivos do projeto, que incluíam a avaliação da exposição a dez fatores de risco à saúde, dentre os quais os indicadores de saúde mental.

Para o cálculo do tamanho da amostra foram utilizados os seguintes critérios: população estimada em 353 mil sujeitos; intervalo de confiança de 95\%; erro amostral de três pontos percentuais; prevalência estimada em $50 \%$; e efeito de delineamento amostral estabelecido em quatro vezes o tamanho mínimo da amostra. Isto representaria uma amostra mínima com 


\section{7 sujeitos.}

Procurou-se garantir que a amostra selecionada representasse a população alvo quanto à distribuição conforme região geográfica, porte das escolas e período de matrícula dos estudantes (diurno e noturno). A distribuição regional foi observada pelo número de escolas existentes em cada uma das 17 GEREs (Gerências Regionais de Ensino). A fim de subsidiar o planejamento amostral, as escolas foram classificadas em três categorias: pequeno porte (menos de 200 alunos); médio porte (200 a 499 alunos); e grande porte (500 alunos ou mais). Alunos matriculados no período da manhã e da tarde foram agrupados numa única categoria (estudantes do período diurno).

Para seleção da amostra requerida, recorreu-se a um procedimento de amostragem por conglomerados em dois estágios, sendo que a "escola" e a "turma" representaram as unidades amostrais, respectivamente, no primeiro e no segundo estágio. Todas as escolas da rede pública estadual em Pernambuco foram consideradas elegíveis para inclusão no estudo. No primeiro estágio, adotou-se como critério de estratificação para realização do sorteio a densidade de escolas em cada microrregião do Estado (GEREs) por porte. No segundo estágio, considerouse a densidade de turmas nas escolas sorteadas por período (diurno e noturno) como critério para sorteio das turmas nas quais os questionários seriam aplicados. Todos os estudantes das turmas sorteadas foram convidados a participar do estudo. Os sorteios foram realizados mediante geração de número aleatórios através do programa EpiInfo (versão 6.04d).

A coleta dos dados foi realizada no período de abril a outubro de 2006. A aplicação dos questionários foi efetuada por seis mestrandos do Programa de Pós-graduação em Hebiatria da Universidade de Pernambuco, que participaram de uma capacitação prévia para padronização dos procedimentos de coleta de dados. A aplicação dos questionários foi efetuada em sala de aula, sem a presença dos professores da escola, para todos os estudantes convidados independentemente da idade. Foi obtido o termo de consentimento negativo (parental passive consentform) dos pais ou responsáveis pelos estudantes menores de 18 anos e diretamente no caso dos estudantes com 18 anos ou mais. Além disso, os questionários não continham qualquer tipo de identificação pessoal (nome, matrícula). Os alunos foram continuamente assistidos pelos aplicadores (sempre dois por turma) para que pudessem esclarecer dúvidas e auxiliar no preenchimento das informações.
O questionário utilizado para obtenção dos dados relativos às variáveis foi uma versão traduzida, autoadministrada e previamente testada do Global School-based Student Health Survey (GSHS), proposto pela Organização Mundial da Saúde (OMS), disponível para consulta no seguinte endereço eletrônico www.who.int/chp/gshs/en. O questionário é composto por dez módulos: características pessoais; consumo de álcool e drogas; hábitos alimentares; higiene; saúde mental; atividades físicas; comportamentos na escola; comportamento sexual; tabagismo; e violência. A utilização de questionários em estudos epidemiológicos com foco na análise de indicadores de saúde mental é um procedimento metodológico que vem sendo adotado em investigações congêneres ${ }^{14-17}$ devido ao baixo custo e facilidade de aplicação.

Previamente ao inicio da coleta de dados, um estudo piloto foi conduzido a fim de determinar indicadores de reprodutibilidade de medidas e testar a aplicabilidade do instrumento. Os dados para o estudo piloto foram coletados em duas escolas da rede pública municipal de ensino na cidade do Recife, com uma amostra de 138 adolescentes com idade de 14 a 19 anos (59 moças). Indicadores de reprodutibilidade (consistência de medidas testereteste) foram de moderados a altos na maioria dos itens do instrumento, sendo que os coeficientes de concordância (índice kappa) para medidas dos indicadores de saúde mental variaram de 0,60 (qualidade do sono) a 0,93 (pensamento de suicídio).

Cinco indicadores de saúde mental foram analisados neste estudo e definidos operacionalmente como variáveis dependentes (sentimento de solidão, dificuldade para dormir devido à preocupação, sentimento de tristeza, pensamento de suicídio e planos de suicídio). Sentimento de solidão foi determinado mediante análise das respostas à questão "durante os últimos 12 meses, com que frequência você se sentiu sozinho?", sendo que todos os sujeitos que referiram "a maioria das vezes" ou "sempre" foram classificados como casos. Similarmente, dificuldade para dormir devido à preocupação foi identificada pela resposta à pergunta "durante os últimos 12 meses, com que frequência você esteve tão preocupado com alguma coisa que não conseguiu dormir à noite?", sendo que os sujeitos que responderam "a maioria das vezes" ou "sempre" foram classificados como casos. Casos de sentimento de tristeza foram identificados por respostas afirmativas à questão "durante os últimos 12 meses, você se sentiu 'muito triste' ou 'sem esperança' quase todos os dias durante duas semanas ou mais seguidas, a ponto de você ter que parar de fazer suas atividades normais?”. 
Pensamento de suicídio foi identificado pela resposta afirmativa dos sujeitos à pergunta "durante os últimos 12 meses você pensou em se suicidar?”. Na variável plano de suicídio, os casos foram identificados pela resposta afirmativa dos sujeitos à pergunta "durante os últimos 12 meses você fez planos para se suicidar?”.

A descrição de cada uma das perguntas utilizadas e do modo de classificação dos adolescentes a partir das respostas referidas pelos mesmos, justifica-se porque a redação das perguntas em português pode não corresponder a uma tradução literal do original em inglês. Além disso, a pergunta utilizada para obter um indicador de pensamento em suicídio ("durante os últimos 12 meses você pensou em se suicidar?") pode representar um indicador de saúde mental de menor gravidade do que aquele avaliado em estudos no qual a versão original em inglês da pergunta foi utilizada ("During the past 12 months, did you ever seriously consider attempting suicide?").

Para fins de análise, as variáveis independentes foram subdivididas em: demográficas (sexo, faixa etária e cor da pele/etnia); socioeconômicas (morar com os pais, local de residência, escolaridade materna e situação ocupacional do adolescente); e relacionadas à escola (turno e série). A idade foi dicotomizada nas seguintes faixas etárias: $14-16 \mathrm{e}$ 17-19 anos. A cor da pele/etnia foi auto-referida considerando seis possíveis respostas (branca, preta, parda, indígena, amarela, outra) e, em seguida, agrupada em duas categorias (branco, não branco). A variável "morar com os pais" foi dicotômica ( $\operatorname{sim} /$ não) e somente os estudantes que referiram morar com pai e mãe foram classificados na categoria "sim". O local de residência foi referido em duas categorias (urbano, rural). A escolaridade materna foi referida pelo filho e classificada em três categorias ( $\leq 8$ anos, 9-11 anos, 12 anos ou mais de estudo). A situação ocupacional do adolescente foi uma variável dicotômica (trabalha/não trabalha), analisada mediante engajamento do adolescente no mercado de trabalho formal ou informal; assim, todos que relataram trabalhar, independente de ter ou não carteira profissional assinada, foram classificados como trabalhadores. O turno em que estudam foi agrupado em diurno (abrangendo aqueles que estudam no período matutino e vespertino) e noturno. A série escolar foi codificada como padronizado no sistema educacional.

A tabulação dos dados foi efetuada no programa EpiData, utilizando-se controles automáticos de amplitude e consistência na entrada dos dados. Os dados foram digitados em duplicata sendo utilizada a ferramenta "validação de dupla digitação" a fim de detectar e corrigir possíveis erros de digitação.

Análises estatísticas foram realizadas mediante utilização do programa SPSS para Windows (versão 10). Análises descritivas foram efetuadas por distribuição de frequências. Para análise da associação entre variáveis, recorreu-se à aplicação do teste de qui-quadrado de Pearson e, no caso das variáveis em escala ordinal, ao qui-quadrado para tendência.

Visando identificar fatores demográficos, socioeconômicos e escolares associados a cada um dos cinco indicadores de saúde mental (desfechos), recorreu-se à análise de regressão logística binária e em seguida multivariada adotando método backward stepwise. Todas as variáveis independentes foram incluídas no modelo inicial e então foram regressivamente excluídas até que somente aquelas com valor $p$ inferior a 0,05 foram mantidas.

\section{Resultados}

Foram visitadas 76 escolas ( $11 \%$ do total de escolas estaduais do Estado) em 44 municípios, o que representa $23 \%$ do total de municípios Pernambucanos. Foram entrevistados 4.210 estudantes com idade entre 14 e 19 anos (59,8\% do sexo feminino), sendo que 83 sujeitos que foram convidados a participar do estudo se recusaram ( $1,9 \%$ de recusas). O detalhamento das características demográficas e socioeconômicas dos sujeitos está apresentado na Tabela 1.

A prevalência de "sentimento de solidão" foi de 15,8\% (IC95\%:15,3;16,1), significativamente maior entre as moças $(19,6 \%)$ em comparação aos rapazes $(11,8 \%)$. Ao analisar associação desta variável com os fatores demográficos, socioeconômicos e escolares, identificou-se que morar com os pais e o turno de matricula na escola discriminaram significativamente a ocorrência deste desfecho. A prevalência de solidão foi maior entre os adolescentes que não moram com os pais e entre aqueles que estudam no período noturno (Tabela 2). Ao estratificar estas análises por gênero, verificou-se que os resultados permanecem inalterados, exceto pela associação entre sentimento de solidão e turno que foi identificada somente entre as moças.

Faixa etária, turno e morar com os pais foram fatores que apresentaram também associação com a "dificuldade para dormir devido à preocupação" (Tabela 2). A prevalência desta condição na amostra total foi de $10,0 \%$ (IC95\%: 8,5;11,0), sendo maior entre as moças ( $14,6 \%$ versus $6,5 \%)$, os adolescentes na faixa etária de 17 a 19 anos, os sujeitos que não moram com a família e entre aqueles que estudam 
Tabela 1

\begin{tabular}{|c|c|c|c|c|c|c|}
\hline \multirow[t]{2}{*}{ Variável } & \multicolumn{2}{|c|}{ Rapazes } & \multicolumn{2}{|c|}{ Moças } & \multicolumn{2}{|c|}{ Total } \\
\hline & $\mathrm{n}$ & $\%$ & $\mathrm{n}$ & $\%$ & $\mathrm{n}$ & $\%$ \\
\hline \multicolumn{7}{|l|}{ Idade (anos) } \\
\hline 14 & 51 & 3,0 & 135 & 5,4 & 186 & 4,4 \\
\hline 15 & 203 & 12,0 & 443 & 17,6 & 646 & 15,4 \\
\hline 16 & 344 & 20,4 & 587 & 23,4 & 931 & 22,2 \\
\hline 17 & 497 & 29,5 & 611 & 24,3 & 1108 & 26,4 \\
\hline 18 & 366 & 21,7 & 460 & 18,3 & 826 & 19,7 \\
\hline 19 & 226 & 13,4 & 275 & 11,0 & 501 & 11,9 \\
\hline \multicolumn{7}{|l|}{ Cor da pele/etnia } \\
\hline Branco & 417 & 24,8 & 639 & 25,5 & 1056 & 25,2 \\
\hline Não branco & 1262 & 75,2 & 1866 & 74,5 & 3128 & 74,8 \\
\hline \multicolumn{7}{|l|}{ Mora com os pais } \\
\hline $\operatorname{Sim}$ & 1130 & 68,1 & 1510 & 60,6 & 2640 & 63,6 \\
\hline Não & 529 & 31,9 & 981 & 39,4 & 1510 & 36,4 \\
\hline \multicolumn{7}{|c|}{ Local de residência } \\
\hline Urbana & 1311 & 78,1 & 1983 & 79,5 & 3294 & 79,0 \\
\hline Rural & 367 & 21,9 & 510 & 20,5 & 877 & 21,0 \\
\hline \multicolumn{7}{|c|}{ Escolaridade materna (anos) } \\
\hline$\leq 8$ & 1086 & 69,4 & 1771 & 74,5 & 2857 & 72,5 \\
\hline $9-11$ & 352 & 22,5 & 480 & 20,2 & 832 & 21,1 \\
\hline$\geq 12$ & 127 & 8,1 & 126 & 5,3 & 253 & 6,4 \\
\hline \multicolumn{7}{|c|}{ Situação ocupacional } \\
\hline Não trabalha & 1157 & 69,2 & 2119 & 84,8 & 3276 & 78,5 \\
\hline Trabalha & 514 & 30,8 & 381 & 15,2 & 895 & 21,5 \\
\hline \multicolumn{7}{|l|}{ Turno } \\
\hline Diurno & 908 & 53,9 & 1506 & 60,0 & 2414 & 57,6 \\
\hline Noturno & 778 & 46,1 & 1002 & 40,0 & 1780 & 42,4 \\
\hline \multicolumn{7}{|c|}{ Série do ensino médio } \\
\hline $1^{\circ}$ ano & 778 & 46,1 & 1099 & 43,8 & 1877 & 44,7 \\
\hline $2^{\circ}$ ano & 522 & 31,0 & 818 & 32,6 & 1340 & 31,9 \\
\hline $3^{\circ}$ ano & 386 & 22,9 & 593 & 23,6 & 979 & 23,3 \\
\hline
\end{tabular}

em período noturno. As análises estratificadas por gênero evidenciaram que a associação com a faixa etária permanece inalterada; entretanto, a associação entre dificuldade para dormir devido à preocupação com as variáveis turno e morar com os pais foi significativa somente entre as moças.

Rapazes e moças também diferiram quanto à prevalência de sentimento de tristeza que foi, respectivamente, de $25,4 \%$ e $35,6 \%$. Na amostra em geral, a prevalência foi de 29,7\% (IC95\%:27,9;31,1). Identificou-se que todas as variáveis independentes com exceção da "cor da pele/etnia" estavam significativamente associadas à ocorrência deste evento. A prevalência de sentimento de tristeza foi maior entre os adolescentes: mais velhos (17-19 anos), que não moram com os pais, residentes em área urbana, filhos de mãe com baixa escolaridade, que trabalham, que estudam no período noturno e entre aqueles matriculados na primeira série do ensino médio (Tabela 2). Ao estratificar as análises, verificou-se que, independente do gênero, a prevalência de sentimento de tristeza foi significativamente maior entre aqueles que não moram com os pais, filhos de mãe com baixa escolaridade e matriculados no período noturno.

Faixa etária, cor da pele, local de residência, turno e morar com os pais foram fatores que apresentaram também associação com pensamento de suicídio (Tabela 2). A prevalência desta condição na amostra total foi de 11,2\% (IC95\%: 11,7;10,9), 


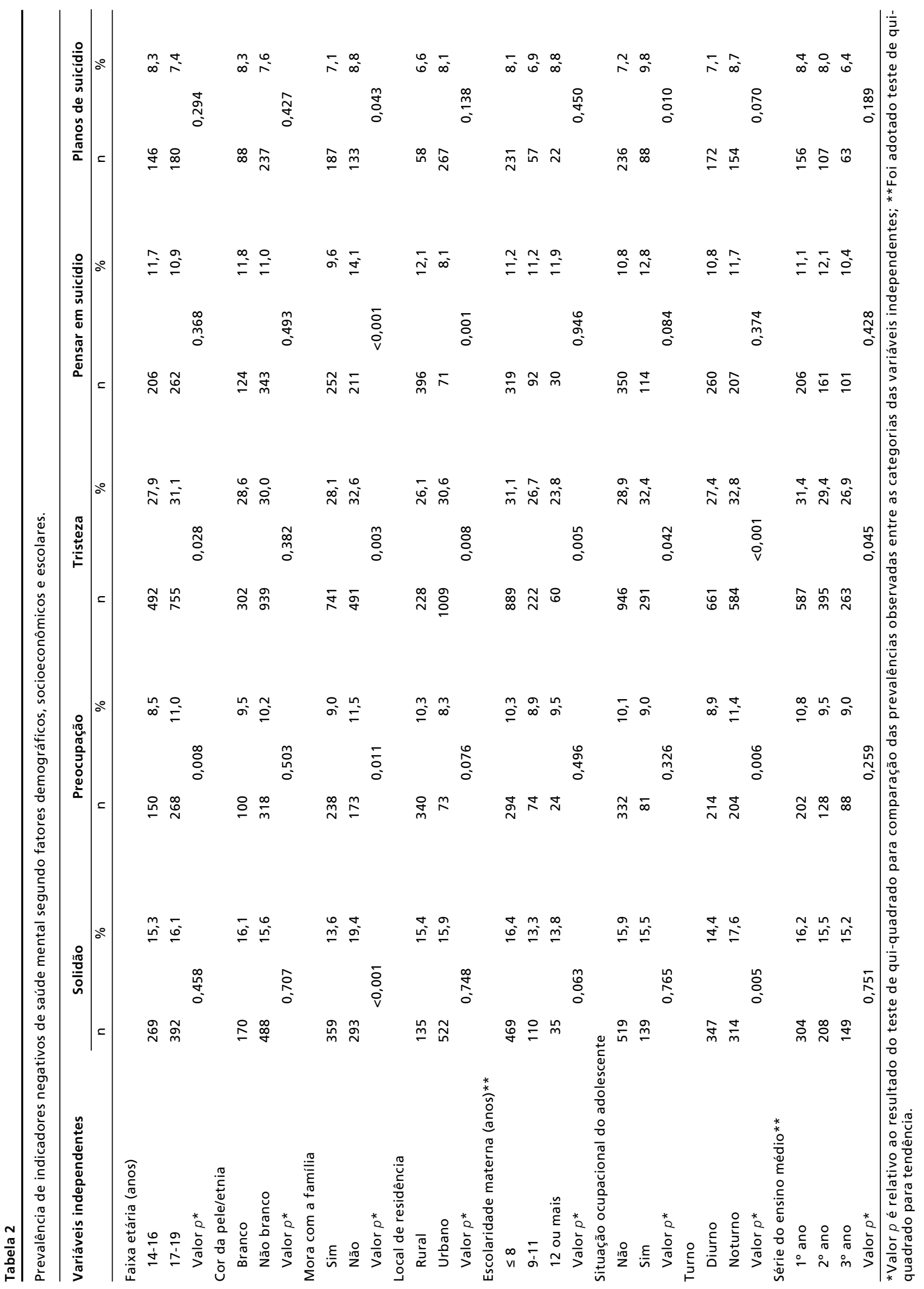


sendo maior entre os adolescentes na faixa etária de 14 a 16 anos, entre os sujeitos que não moram com os pais e entre aqueles que estudam em período noturno. As análises estratificadas por gênero evidenciaram que a associação com a faixa etária permanece inalterada; entretanto, a associação entre a ocorrência de pensamento de suicídio com as variáveis "situação ocupacional" e "morar com os pais" foi significativa somente entre as moças.

A prevalência de planos de suicídio na amostra foi de 7,8\% (IC95\%: 8,3;7,4). Analisando a associação desta variável com os fatores independentes considerados neste estudo, identificou-se que morar com os pais e situação ocupacional do adolescente discriminaram significativamente a ocorrência deste evento. A prevalência de planos suicídios foi maior entre os adolescentes que declararam não residir com os pais e entre aqueles que trabalham (Tabela 2). Ao estratificar estas análises por sexo, verificou-se que a escolaridade materna, entre os rapazes, e a situação ocupacional, entre as moças, foram as únicas variáveis associadas à ocorrência deste evento

Independente do sexo, a variável "morar com os pais" apresentou associação significativa com sentimento de solidão e de tristeza. Entre os rapazes, o valor $p$ observado $(p=0,069)$ na análise de associação entre as variáveis "morar com a família" e "pensamento em suicídio" foi discretamente superior ao previamente estabelecido (Tabela 3 ).

A regressão logística não ajustada (Tabela 4) e, particularmente, as análises multivariáveis (Tabela 5) permitiram identificar que a cor da pele e a escolaridade materna não foram abordadas como fatores associados a nenhum dos indicadores de saúde mental analisados no presente estudo. Por outro lado, sexo foi um fator que discriminou a prevalência de todos os indicadores, observando-se maior chance de referir indicadores negativos de saúde mental entre as moças em comparação aos rapazes. Estudantes mais velhos (17-19 anos) em comparação aos mais jovens (14-16 anos) apresentaram maior chance de relatar dificuldade de dormir devido à preocupação e sentimento de tristeza.

Residir em área urbana foi identificado como fator de proteção para dificuldade de dormir devido à preocupação, sentimento de tristeza e pensamento de suicídio. A chance de adolescentes residentes em área urbana relatarem dificuldade de dormir devido à preocupação, sentimento de tristeza e pensamento de suicídio foi, respectivamente, $26 \%, 27 \%$ e $33 \%$ inferior em comparação aos residentes em área rural.

Não morar com os pais e estudar no período noturno foram identificados como fatores que elevam a chance de "sentimento de solidão". Além disso, estudar no período noturno foi identificado também como fator diretamente associado a sentimento de tristeza.

A situação ocupacional do estudante também foi identificada como fator associado a indicadores negativos de saúde mental. Observando-se que aqueles estudantes que referiram trabalhar apresentaram maior chance de referirem sentimento de tristeza, pensamento de suicídio e planos de suicídio.

\section{Discussão}

Os resultados deste estudo permitiram evidenciar alguns importantes achados: a) tanto em rapazes quanto em moças o indicador sentimento de tristeza foi o de maior prevalência neste subgrupo populacional; b) a frequência dos cinco indicadores negativos de saúde mental analisados neste estudo foi maior nas moças; c) análises multivariáveis evidenciaram que a cor da pele e a escolaridade materna não são fatores associados à ocorrência dos indicadores negativos de saúde mental; d) os estudantes do período noturno apresentam maior chance de referir sentimento de solidão e sentimento de tristeza; e, e) os estudantes residentes em áreas urbanas apresentaram menor chance de relatarem três indicadores negativos de saúde mental (dificuldade de dormir devido à preocupação, sentimento de tristeza e pensamento em suicídio).

No entanto, algumas limitações precisam ser consideradas neste estudo. Trata-se de estudo transversal, onde viés de causalidade reversa é uma característica inerente. Portanto, deve-se interpretar com cautela o sentido das associações que foram identificadas neste estudo. A amostra não abrangia os adolescentes que estão matriculados na rede privada de ensino e aqueles que estão fora do sistema escolar; por isso, mesmo considerando que o estudo foi desenvolvido com uma amostra relativamente grande, a generalização dos resultados deve ser limitada à população alvo do estudo (adolescentes estudantes do ensino médio da rede pública estadual de ensino).

Apesar das limitações, esta investigação apresenta pontos positivos que merecem ser destacados, como: a abrangência da pesquisa (base estadual), o dimensionamento amostral que garante precisão às análises e a inclusão de estudantes residentes em área urbana e rural. Outro ponto positivo diz respeito à utilização de um questionário que foi previamente testado e apresentou boa consistência de medidas (reprodutibilidade), assim como o fato deste instrumento ter sido aplicado por equipe qualificada e treinada para este fim. 


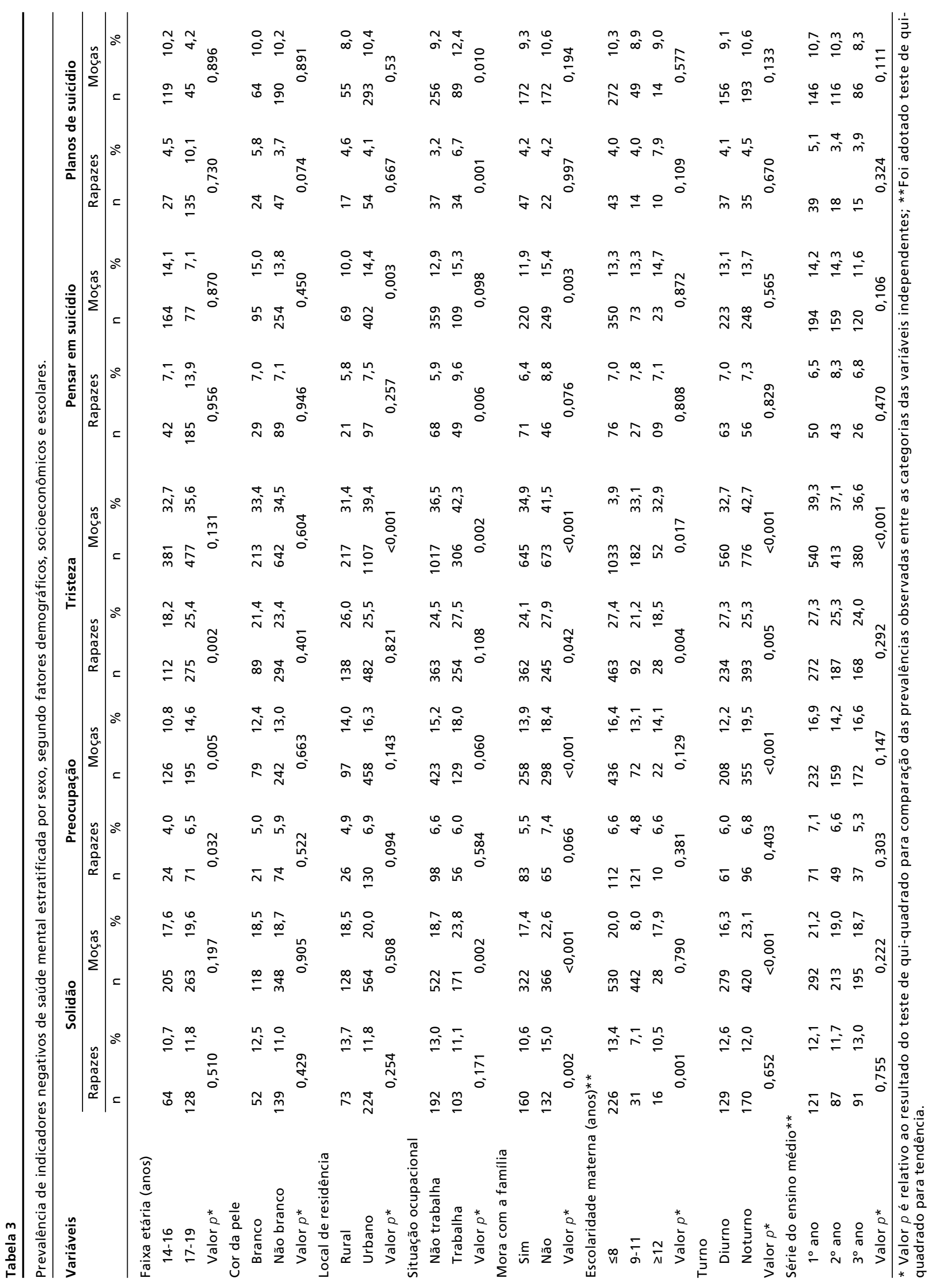




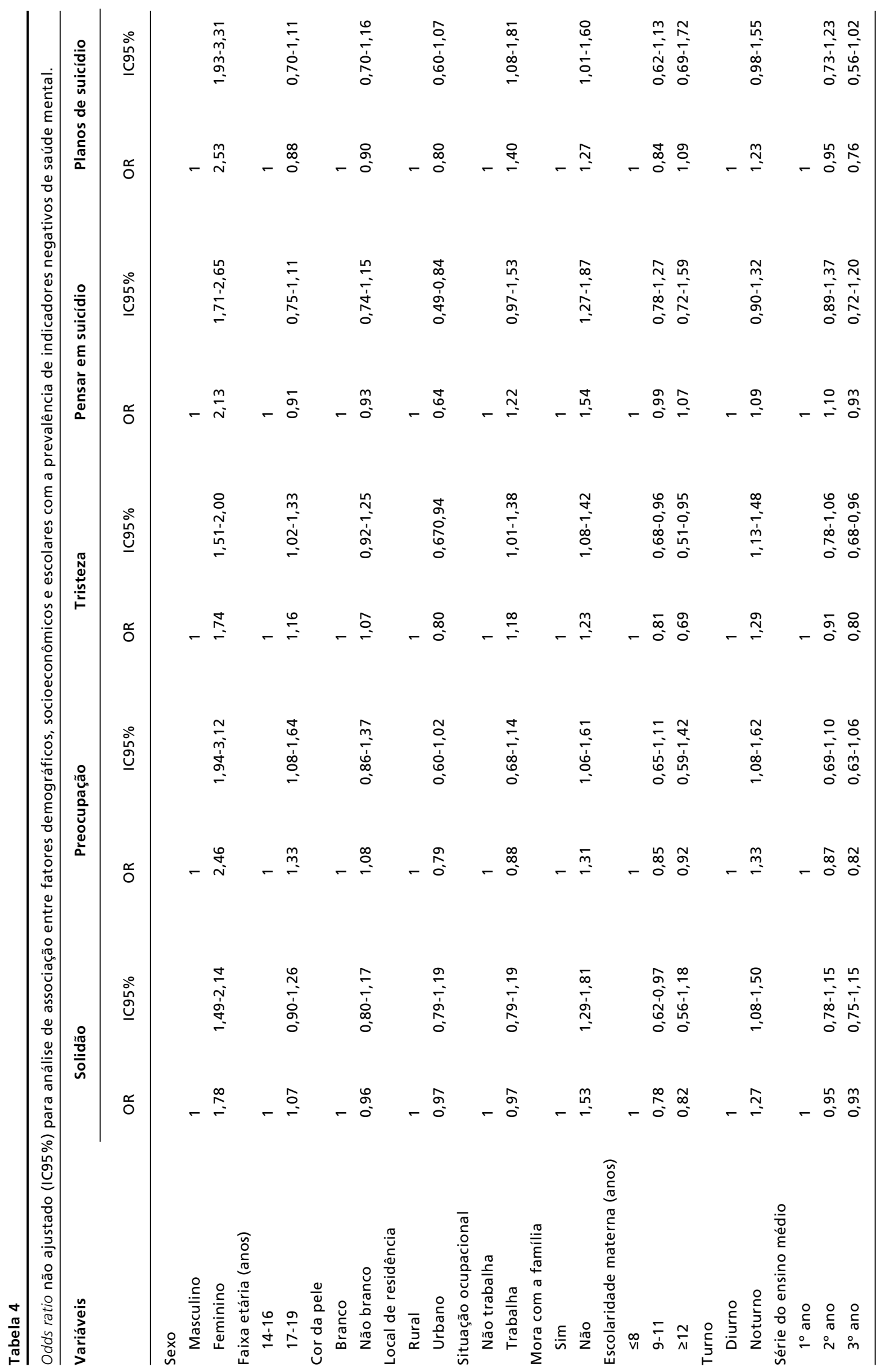




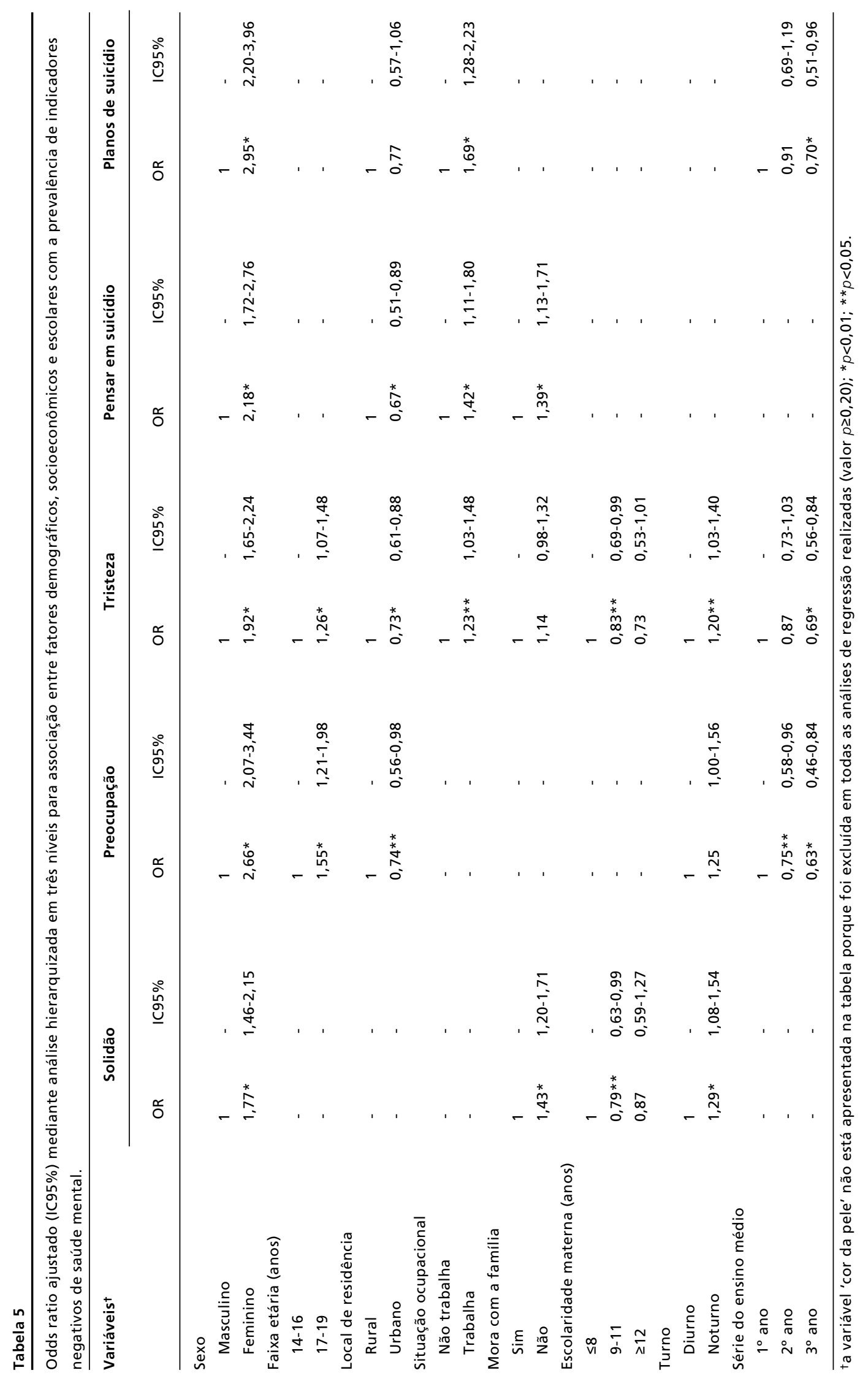


Tristeza foi o indicador de saúde mental de maior prevalência neste subgrupo populacional. Este achado é consistente com os resultados relatados por Mahfoud et al. 15 e Peltzer ${ }^{17}$ que estudaram, respectivamente, adolescentes libaneses e africanos. Foram localizados dois estudos 16,18 que identificaram a dificuldade de dormir devido à preocupação como o fator com maior prevalência e, ainda, outro ${ }^{13}$ no qual as prevalências observadas para os diversos indicadores de saúde mental analisados foram praticamente as mesmas. O componente metodológico não parece ser a explicação para esta discrepância nos resultados visto que todos os estudos referenciados adotaram o mesmo instrumento de coleta de dados (GSHS), e os mesmos procedimentos para dimensionamento e seleção amostral. As variações observadas então poderiam ser, ao menos em parte, explicadas pelo modo como os sujeitos reagem aos diferentes agentes estressores aos quais estão expostos.

Observou-se no presente estudo uma maior prevalência de indicadores negativos de saúde mental entre as moças. Este resultado permaneceu inalterado após realização de análises multivariáveis e é consistente com as evidências relatadas em investigações congêneres.16-19 No estudo de Brown et al. 20 conduzido com adolescentes africanos, não foi observada diferença entre rapazes e moças quanto a prevalência de sentimento de solidão; curiosamente Peltzer17 também investigando indicadores de saúde mental em adolescentes africanos verificou diferença estatisticamente significativa entre rapazes $(15,4 \%)$ e moças $(16,7 \%)$ quanto ao sentimento de solidão, mas não encontrou diferenças entre rapazes e moças quanto à prevalência de dificuldade de dormir devido à preocupação.

É possível que esta diferença observada entre rapazes e moças quanto à prevalência de indicadores de saúde mental seja decorrente de fatores culturais. Por exemplo, mulheres mesmo na adolescência podem ter maior facilidade que os rapazes para reconhecerem sentimentos de tristeza, solidão ou desesperança. Há de se considerar também a possibilidade de que o contexto sociocultural onde o estudo foi realizado possa conduzir os rapazes a não referir a presença destes indicadores de saúde mental, uma hipótese que precisará ser investigada no futuro, possivelmente mediante abordagens qualitativas de investigação.

Além do gênero, outros fatores demográficos e socioeconômicos podem estar associados à saúde mental em adolescentes, mas há escassez de dados na literatura e poucas variáveis explanatórias (idade, tipo de escola) foram investigadas. Por exemplo, os estudos de Mahfoud et al.15 e Rudatskira et al.18 apresentaram evidências convergentes em relação aos resultados do presente estudo de que a idade não é um fator associado a pensamento de suicídio em adolescentes. De modo divergente, os resultados relatados por Cheng et al. ${ }^{19}$ revelaram um aumento da prevalência de pensamento em suicídio com aumento da idade em adolescentes chineses.

No presente estudo, independente do sexo, a variável morar com os pais apresentou associação significativa com sentimento de solidão e pensamento em suicídio, mas não com os demais indicadores de saúde mental. Os resultados são divergentes em relação à evidência apresentada por Borges et al. ${ }^{21} \mathrm{em}$ seu estudo com adolescente mexicanos, segundo o qual viver com os pais não foi um fator associado nem à variável pensamento em suicídio nem a variável planos de suicídio.

A identificação de que os estudantes do período noturno apresentam maior chance de referir sentimento de solidão e sentimento de tristeza constitui um dos mais importantes achados desta investigação. Diferente do que ocorre em países desenvolvidos, no Brasil, mais de $50 \%$ de todos os estudantes estão matriculados no período noturno e evidências disponíveis sugerem que estes sujeitos constituem um subgrupo vulnerável, com maior prevalência de exposição a fatores comportamentais de risco à saúde. 22

A escola é reconhecidamente um ambiente favorável ao desenvolvimento de ações de promoção à saúde, não apenas porque é possível atingir um grande número de sujeitos, mas principalmente porque estudos de revisão vêm evidenciando que intervenções neste ambiente tendem a ser mais eficientes e efetivos. 23,24 Apesar disto, este contexto de intervenção continua ainda pouco explorado nas políticas públicas de saúde, em especial ao considerar as políticas de atenção à saúde mental.

Os resultados apresentados neste estudo sugerem que programas são necessários para reduzir a prevalência de indicadores negativos de saúde mental em adolescentes estudantes do ensino médio. Além disso, moças e estudantes do período noturno parecem constituir os subgrupos mais vulneráveis e que, portanto, deveriam ser priorizados na impossibilidade de desenvolvimento de intervenções direcionadas a todos os estudantes. As informações relatadas neste estudo, acredita-se, podem orientar o planejamento e a avaliação de ações sanitárias com melhor alocação dos recursos disponíveis.

Outras investigações são necessárias a fim de identificar fatores comportamentais associados à ocorrência destes indicadores negativos de saúde mental, como o tabagismo, o consumo de bebidas 
alcoólicas e uso de drogas. Além disso, o estudo de intervenções para redução da prevalência destes indicadores constitui também uma prioridade,

\section{Referências}

1. World Health Organization. World Health Report 2002. Geneva, Switzerland; 2002.

2. World Health Organization. Global Status Report on Alcohol. Geneva, Switzerland; 2004.

3. Poikolainen K, Tuulio-Henriksson A, Aalto-Setala T, Marttunen M, Lonnqvist J. Predictors of alcohol intake and heavy drinking in early adulthood: a 5-year follow-up of 15-19 year-old Finnish adolescents, Alcohol and Alcohol. 2001; 36: 85-8.

4. Zhang J, Vitaliano PP, Lin HH. Relations of caregiving stress and health hepend on the health indicators used and gender. Int J Behav Med. 2006; 13: 173-81.

5. Panter-Brick C, Eggerman M, Mojadidi A, Mcdade TW. Social stressors, mental health, and physiological stress in an urban elite of young Afghans in Kabul. Am J Hum Biol. 2008; 20: 627-41.

6. Page RM, Zarco EP, Ihasz F, Suwanteerangkul J, Uvacsek M, Mei-Lee C, Miao NF, Simonek J, Klarova R, Hantiu I, Kalabiska I. Cigarette smoking and indicators of psychosocial distress in Southeast Asian and Central-Eastern European adolescents. J Drug Educ. 2008; 38: 307-28.

7. Fagg J, Curtis S, Stansfeld S, Congdon P. Psychological distress among adolescents, and its relationship to individual, family and area characteristics in East London. Soc Sci Med. 2006; 63: 636-48.

8. Wissow LS, Wilson ME, Roter D, Larson S, Berman H. Family violence and the evaluation of behavioral concerns in a pediatric primary care clinic. Med Care. 1992; 30(Suppl. 5): 150-65

9. Peltonen K, Ellonen N, Larsen HB, Helweg-Larsen K. Parental violence and adolescent mental health. Eur Child Adolesc Psychiatry. 2010; 19: 813-22.

10. Due P, Holstein BE, Soc MS. Bullying victimization among 13 to 15 -year-old school children: results from two comparative studies in 66 countries and regions. Int J Adolesc Med Health. 2008; 20: 209-21.

11. Page RM, Hall CP. Psychosocial distress and alcohol use as factors in adolescent sexual behavior among sub-Saharan African adolescents. J Sch Health. 2009; 79: 369-79.

12. Bezerra J, Barros MV, Tenório MC, Tassitano RM, Barros SS, Hallal PC. Religiousness, alcohol consumption and smoking in adolescence. Rev Panam Salud Publica. 2009; 26: 440-6

13. Cheng Y, Newman IM, Qu M, Mbulo L, Chai Y, Chen Y, Shell DF. Being bullied and psychosocial adjustment among middle school students in China. J Sch Health. 2010; 80: 193-9.

Recebido em 19 de janeiro de 2011

Versão final apresentada em 30 de maio de 2011

Aprovado em 21 de junho de 2011 considerando-se a escassez de evidências sobre estratégias efetivas para lidar com estes eventos de saúde.
14. Xiong GL, Wu J, Shen QY, Mo SX, Yang DW, Zhang QY, Zhang P. Survey on the epidemic characteristics of suicidal tendency among middle-school students in cities. Zhonghua Liu Xing Bing Xue Za Zhi. 2007; 28: 127-30.

15. Mahfoud ZR, Afifi RA, Haddad PH, Dejong J. Prevalence and determinants of suicide ideation among Lebanese adolescents: Results of the GSHS Lebanon 2005. J Adolesc. 2011; 34: 379-84.

16. Fleming LC, Jacobsen KH. Bullying and symptoms of depression in chilean middle school students. J Sch Health. 2009; 79: 130-37.

17. Peltzer K. Health behavior and protective factors among school children in four African countries. Int J Behav Med. 2009; 16: 172-80.

18. Rudatsikira E, Muula AS, Siziya S, Twa-Twa J. Suicidal ideation and associated factors among school-going adolescents in rural Uganda. BMC Psychiatry. 2007; 7:67.

19. Cheng Y, Tao M, Riley L, Kann L, Ye L, Tian X, Tian B, $\mathrm{Hu}$ J, Chen D. Protective factors relating to decreased risks of adolescent suicidal behavior. Child Care Health Dev. 2009; 35: 313-22.

20. Brown DW, Riley L, Butchart A, Meddings DR, Kann L, Harvey AP. Exposure to physical and sexual violence and adverse health behaviours in African children: results from the global school-based student health survey. Bull World Health Organ. 2009; 87: 447-55.

21. Borges G, Benjet C, Medina-Mora ME, Orozco R, Familiar I, Nock MK, Wang PS. Service use among Mexico city adolescents with suicidality. J Affect Disord. 2010; 120: 329.

22. Farias Jr JC, Nahas MV, Barros MVG, Loch MR, Oliveira ESA, De Bem MFL, Lopes AS. Comportamentos de risco à saúde em adolescentes no Sul do Brasil: prevalência e fatores associados. Rev Panam Salud Publica. 2009; 25: 344-52.

23. Collins J, Robin L, Wooley S, Fenley D, Hunt P, Taylor J, Haber D, Kolbe L. Programs-that-work; CDC's guide to effective programs that reduce health-risk behavior of youth. J Sch Health. 2002; 72: 93-9.

24. Hoehner CM, Soares J, Parra Perez D, Ribeiro IC, Joshu CE, Pratt M, Legetic BD, Malta DC, Matsudo VR, Ramos LR, Simões EJ, Brownson RC. Physical activity interventions in Latin America: a systematic review. Am J Prev Med. 2008; 34: 224-33. 